\title{
Editorial
}

\section{Celebrating 30 years of NeuroRehabilitation}

In 2021 NeuroRehabilitation (NRE) celebrated its $30^{\text {th }}$ anniversary, and there was much to celebrate. The journal has been led for 30 years by its internationally renowned Editors-in-Chief Nathan D. Zasler, MD, and Jeffrey S. Kreutzer, PhD. They are supported by their invaluable managing editor, Melissa Oliver, OTR for the last 15 years, as well as an excellent multidisciplinary editorial board. In its 30-year history the journal has seen several great achievements. Possibly the most important of these is pioneering an interdisciplinary approach, reflecting the belief of the Editors-in-Chief that the most effective rehabilitation is for multiple disciplines to serve patients' needs in a coordinated fashion, which has helped transform care in this vitally important and highly challenging field.

In support of this aim, NRE has published over 100 extremely valuable thematic issues on a variety of topics (for the full list, see: https://iospress.com/nrespecial-issues). The journal's Impact Factor has also continued its upward trajectory and is currently 2.138 , according to Journal Citation Reports (Clarivate, 2021). Since 2018, NRE and Cochrane Rehabilitation have been affiliated with the aim of improving evidence-based neurorehabilitation practice, through the section in NRE called Cochrane Corners. Each issue contains a summary of Cochrane Reviews and accompanying commentaries that are exclusive to NRE (for the full list, see: https://iospress.com/nrecochrane-corners).

Below you'll find the pictures of the editorial team, as well as an interview with the Editors-in-Chief, which we hope gives the reader an interesting insight into NRE's past, present, and future.

We would like to thank the Editors-in-Chief for their dedication and enormous amount of time and effort spent to make the journal a success. We furthermore would like to thank all authors, editorial board members, reviewers, and readers for their contributions to and support of the journal over the years. We look forward to the next 30 years and appreciate your continuing support for the journal, which makes us optimistic that the fortunes of the journal will continue to grow.

The Publisher IOS Press

\section{Interview with the Editors-in-Chief}

Given the competitive nature of publishing, what was the rationale for launching the journal 30 years ago?

Research on rehabilitation was increasing and most existing publications focused on acute care medical concerns. Professionals, patients, and their families recognized the limits of ongoing neurorehabilitation practice and raised concerns about the efficacy of current treatments. NRE was designed to guide clinical practice via empirical research and keep clinicians aware of the latest evidence-based practice in neurorehabilitation through both original research and thematic issues guest edited by leaders in the field.

What is the greatest challenge faced by the journal?

NRE publishes manuscripts that address a wide variety of medical and psychosocial issues. Finding peer reviewers with relevant expertise who can turn around constructive reviews in a short time-period can be a significant challenge. 


\section{Editorial office}

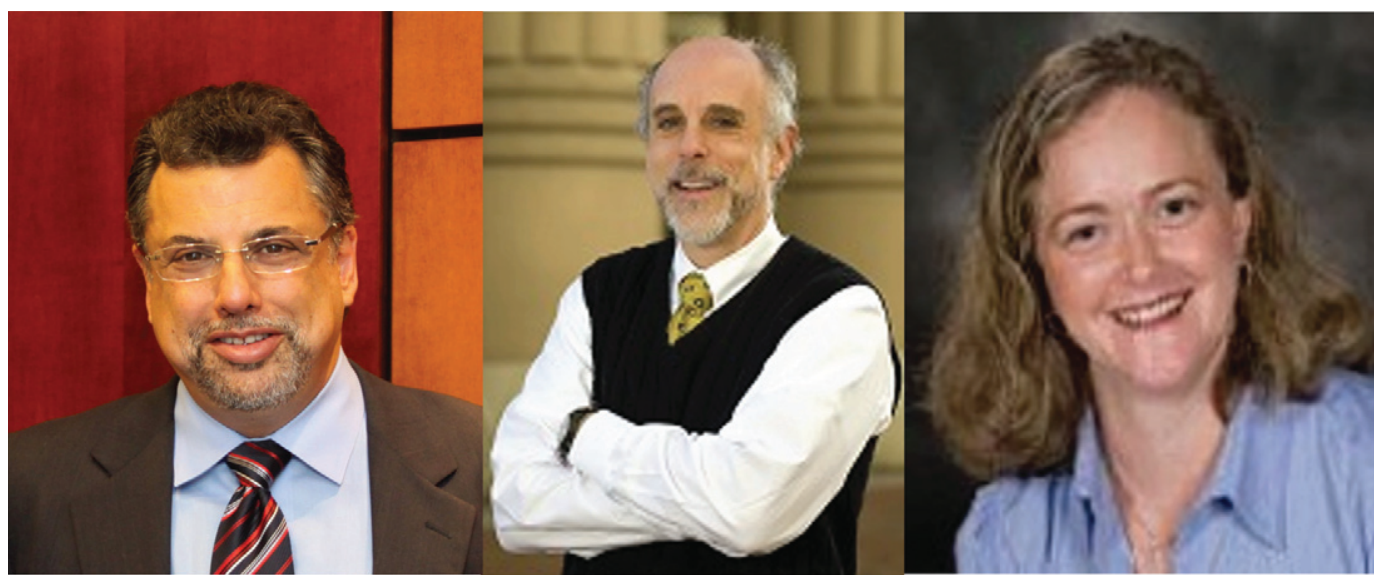

From left to right: Nathan D. Zasler, MD, Jeffrey S. Kreutzer, PhD, and Melissa Oliver

What is unique about the journal's peer review process?

All manuscripts undergo initial screening by either the Editors-in-Chief or Associate Editors and are peer reviewed by at least two experts.

\section{What makes NRE an ideal repository for high} quality papers in the interdisciplinary and multidisciplinary field of neurorehabilitation?

We are most grateful to have an accomplished and active editorial board and multitude/large number of reviewers who offer critically constructive feedback. The review process gives authors an opportunity to improve the quality of their work and ultimately the reputation of the journal.

\section{How has NRE distinguished itself from other journals in the field?}

NRE has focused on topics relevant to interdisciplinary practice. For example, some of the thematic topics have included chronic pain, vegetative state, telerehabilitation, electrical injuries, hypoxic ischemic brain injury, multiple sclerosis, and cognitive prosthetic systems.
What are the most important accomplishments of the past 10 years?

The journal has published manuscripts relevant to a broad readership with cutting edge information directly translatable to clinical practice. Additionally, the journal has become a repository for important clinical information pertinent to the practice of neurorehabilitation regardless of one's specialty. Lastly, the success of the journal can be measured to a great extent by the broad international representation of authors and further affirmed by a continued upward trend of the journal's Impact Factor.

What is the journal's goal for the next 10 years and beyond?

The primary goal is to provide empirically based information relevant to an interdisciplinary group of readers. Ultimately, our goal is to continue to be a source of the best information on current neurorehabilitation science and practice in order to provide a more comprehensive foundation for the field at large. 